\title{
AUDIO-VISUAL DISTRACTION EFFECT ON HEART RATE IN CHILDREN DURING DENTAL TREATMENT, A RANDOMIZED CLINICAL TRIAL
}

\author{
Sherif Adel Zakhary*, Mahmoud Hamdy Eid ${ }^{* *}$ and Nada Mohamed Wassef ${ }^{* * *}$
}

\begin{abstract}
Background: Dental anxiety is one of the causes of avoiding dental visits in children and is associated with physiological body reactions as increased heart rate. Different behavior management techniques have been used to reduce dental anxiety among them are Tell-show-do technique and audiovisual (AV) distraction.
\end{abstract}

Aim: To determine the effect of audiovisual distraction on heart rate during dental treatment in children.

Subjects and methods: Forty-two patients requiring pulp therapy were randomly allocated to either control group (managed by tell-show-do technique) or test group (managed by audiovisual distraction using virtual reality eyeglasses). Heart rate measures were recorded for all patients before the treatment and every five minutes during the procedure. Results were tabulated and statistically analyzed.

Results: There was a statistically significant difference in heart rate mean values between both study groups. Where AV distraction group showed better results in lowering anxiety.

Conclusions: Audiovisual distraction can be considered an effective method for reducing anxiety during dental treatment and helping the patients enjoy the dental visit.

KEYWORDS: Tell-show-do, behavior management, audiovisual distraction, dental anxiety, heart rate.

\section{INTRODUCTION}

Children are victims of fear and anxiety more than adults since children are exposed to more insecurities in their life from movies, cartoons, school and communication with others. Children are also more difficult to handle within the dental visit because of their inability to express their fear and cope with fearful stimulations such as pain or fearful objects (Vilela \& Ogawa, 2019).

* Pediatric Dentistry Master Degree Student.

** Professor of Pediatric Dentistry and Dental Public Health, Faculty of Dentistry, Cairo University.

*** Lecturer of Pediatric Dentistry and Dental Public Health, Faculty of Dentistry, Cairo University. 
The dental clinic and dental work are considered an unpleasant experience for children with many unknown objects for them. Forty eight percent female pediatric patients and thirty eight percent male pediatric patients experience anxiety which causes them to be uncooperative, anxious, afraid and ask a lot of questions making it difficult for the dentist to perform his dental treatment and waste more time during the dental visit for psychological management (Muppa et al., 2013).

Dental Anxiety nowadays is defined as an aversive emotional state of fear, stress or being afraid during the dental visit. Its main consequence is avoiding the dental visit resulting in bad oral healthcare and escalation of dental disease. Thus it is important for all dental practitioners to consider dental anxiety not only during the dental visit, but also after, to assure that the patient leaving the clinic is satisfied emotionally and motivated for the next visit (Facco \& Zanette, 2017).

The difference between fear and anxiety is controversial, sometimes considered the same. However, it has been stated that if the stimulus is known, then the term fear is used, while if the stimulus is unknown then the term anxiety is used (Grupe \& Nitschke, 2013). Dental anxiety has been ranked fifth among commonly feared environments. Given its high prevalence, it is still expected that patients with dental anxiety avoid their dentist (Carter et al., 2014).

The American academy of pediatric dentistry (AAPD) in 2019, stated that the aim of managing children in the dental visit is not only to handle their fear, but also to provide a friendly environment for the patient and help the dentist to do his job without interruptions and facilitate the dental treatment. It also motivates the child for future dental visits thus improving his oral health care.

Behavior management techniques are mainly divided into two categories, pharmacological methods and non-pharmacological methods. The pharmacological methods are medications and sedation techniques which are used in cases of severe uncooperativeness and phobic dental patients (Wang et al., 2008). Non-pharmacologic methods include different methods as communication skills, relaxation techniques, hypnotherapy, acupuncture, distraction, enhancing control, Tell-Show-Do, systematic desensitization, positive reinforcement.

Tell-Show-Do (TSD) technique is the most commonly used technique in pediatric dentistry. It is a very basic technique where it consists of explaining and demonstrating the procedure to the patient and the instruments used during treatment before application.

Distraction is defined as a thing that prevents someone from concentrating on something else. In the past years, dental practitioners tried several methods to distract a child away from the dental environment. Playing a cartoon on the TV or playing relaxing songs in order to distract their attention away from the dental work. (El-Sharkawi et al., 2012).

Audiovisual distraction (AV) virtual reality (VR) using eyewear glasses is a type of distraction where the child is blinded totally from the surroundings with his ears are plugged to be totally distracted by the show he is watching. VR utilizes advanced technologies to create virtual environments that allow patients to be immersed in an interactive, simulated world. There is no chance for the child to see or hear anything from the surrounding environment and at the same time the child is kept entertained during his dental visit.

Anxiety results in physiological changes in the body including increased heart rate (Goulart et al., 2012) which can be detected by using pulse oximeter. Therefore heart rate can be used as a measurement of anxiety in dental patients (Mitrakul et al., 2015). 
Therefore, the aim of this study was to determine the effect of audiovisual distraction technique using virtual reality eyeglasses compared with Tell-ShowDo technique on heart rate during dental treatment in children.

The hypothesis adopted in this study is a null hypothesis; there is no difference in heart rate during dental treatment between children managed by audiovisual distraction and children managed by tell-show-do technique.

\section{SUBJECTS AND METHODS}

Ethical approval was obtained from the Research Ethics Committee (REC), Faculty of Dentistry, Cairo University. The sample size was estimated based on the results of Aminabadi et al., 2012 using PS program. The type I error was set at 0.05 and power set at 0.8 . The sample size estimated was 42 patients (21 in each group).

\section{Subjects:}

This study is a randomized controlled trial and included children who attended the outpatient clinic of Pediatric Dentistry and Dental Public Health Department, Faculty of Dentistry, Cairo University.

\section{Eligibility criteria}

\section{Inclusion criteria}

- Children aged 5-8 years old.

- Children with decayed teeth requiring pulp therapy.

- Cooperative and potentially cooperative children.

- Apparently healthy children; free from any physical, mental or medical handicapping problem.

- No previous dental experience.

\section{Exclusion criteria}

- Children with visual or hearing impairment.

- Children with disability complicating the dental treatment as trismus, or TMJ problems.
- Children with highly inflamed pulp tissue inducing pain during the dental visit.

\section{Methods:}

After patients' recruitment, they were randomly allocated to either control or test group. The random sequences were generated using www.random.com. The sequence generated 2 codes (A \& B) for control and test group respectively, that were folded eight times and placed in sealed opaque envelopes. After the parent signed the informed consent, the patient chose an envelope to be allocated in either control or test group.

- Pulse Oximeter device was introduced to the child and attached to his index finger and the initial reading (baseline) was recorded.

\section{Grouping of children:}

- Group A (Control Group) (TSD): Twentyone children received Tell-Show-Do technique as a behavior management technique.

Tell: The Patient was introduced to the dental clinic, the dental chair, the instruments and the topical anesthetic gel as tooth sleeping drug in very simple words.

Show: The child was allowed hold the tools and see the water splashes out from the handpiece and press on the chair button to see how it moves.

Do: The job was done exactly as explained to the patient in the same sequence.

\section{- Group B (Test Group) (AV Distraction):} Twenty-one children received audiovisual distraction technique using virtual reality eyeglasses as a behavior management technique.

The child was introduced to the dental clinic and the dental chair and was told that the chair might move now or during cleaning the teeth.

The child was introduced to the device Nibiru 2.10.002 AV eyewear glasses (Guangdong, China) 
with screen size 5 inches and 3D optical resin lens, and a sample of the cartoon was played figure (2). Earphones were also used to avoid hearing any voices and help in distraction.

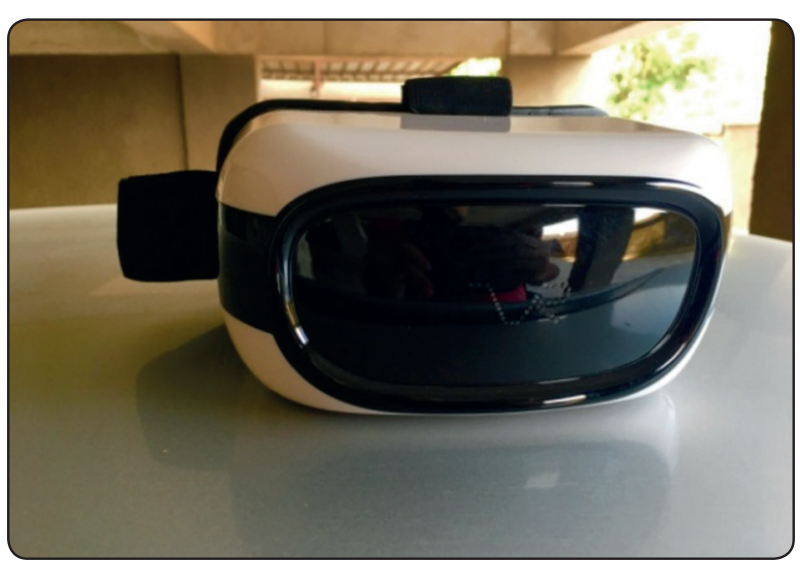

Fig. (2): Nibru AV eyewear glass.

For all patients topical anesthesia gel 20\% benzocaine (Opahl-S, Dharma, USA) was applied, followed by injection of Articane 4\% with epinephrine 1:100,000 (Artinibsa, Inibsa, Barcelona, Spain). Formocresol pulpotomy was performed in all patients, and filling using zinc oxide and eugenol followed by glass ionomer. SSC was done to all cases but not included within the study duration if its time of application exceeded the 20 minutes of the study.

In both groups, heart rate readings of pulse oximeter were recorded every 5 minutes starting from local anesthesia injection till the 20 minutes were reached.

Statistical analysis was performed using IBM SPSS Statistics Version 2.0 for Windows. Data were presented as percentages (\%) for qualitative data as gender; and mean, standard deviation (SD) and median values for quantitative data as age, heart rate. Kolmogorov-Smirnov and Shapiro-Wilk tests were used to assess data normality. The significance level was set at $\mathrm{P} \leq 0.05$.

Age of the participants showed normal distribution, so independent Student-t test was used for in- tergroup comparisons. One-Way ANOVA followed by Bonferroni test were conducted to compare heart rate at different treatment times within the same group. Independent Student-t test (for normally distributed data) and Mann-Whitney U test (for nonnormally distributed data) were used to compare heart rate between both study groups at each treatment time.

\section{RESULTS}

A total of 39 participants were enrolled in this study, whereas 3 were excluded due to technical issues during procedure and nausea from the $\mathrm{AV}$ device. Mean ages of control group $(\mathrm{N}=20)$ was $6.45 \pm 1.23$ years, while in test group $(\mathrm{N}=19)$ mean age was $6.21 \pm 1.18$ with no statistical significant difference. In control group the gender distribution was $11(55 \%)$ males and 9 (45\%) females, while in the test group there were $7(36.84 \%)$ males and $12(63.15 \%)$ females with no statistical significant difference in gender distribution between both study groups. The mean values for heart rate are shown in table (2).

TABLE (2): The mean values for heart rate (beats/ min) of studied groups at different treatment times.

\begin{tabular}{|l|l|l|l|}
\hline & Control & AV Eyewear & P-value \\
\hline Initial reading & $84.0 \pm 5.79$ & $83.57 \pm 5.45$ & $0.810 \mathrm{NS}$ \\
\hline Local anaesthesia & $92.80 \pm 11.08$ & $84.89 \pm 7.77$ & $0.011^{*}$ \\
\hline After 5 mins & $93.10 \pm 9.30$ & $83.57 \pm 5.57$ & $0.000^{*}$ \\
\hline After 10 mins & $91.85 \pm 7.58$ & $82.10 \pm 3.72$ & $0.003^{*}$ \\
\hline After 15 mins & $90.75 \pm 7.61$ & $81.52 \pm 3.84$ & $0.007^{*}$ \\
\hline After 20 mins & $89.9 \pm 5.12$ & $81.42 \pm 2.45$ & $0.025^{*}$ \\
\hline
\end{tabular}

$P \leq 0.05$

*: significant at $P \leq 0.05 ;$ NS: non-significant 


\section{DISCUSSION}

Oral health-care for children is considered a very important factor for maintenance of healthy teeth and gums. There is also a direct connection between oral health and overall health of a patient. Dental anxiety could be an obstacle between children and their oral health-care regarding accepting dental visits (Gupta et al., 2014).

The American Academy of Pediatric dentistry introduced many behavioral management approaches and guidelines in order to assess the patient's degree of anxiety and select the most suitable approach according to the dentist profession and skills. (AAPD, 2019).

The aim of this study was to investigate the effect of Audio-Visual Distraction on the heart rate during the dental visit in order to facilitate the dental visit for both the patient and the dentist. By distracting the patient's eyes and ears, the dentist will save time from introducing his instruments to the child and explaining the noise from the dental handpiece and suction tube, more over the site of the dental needle is blinded by the distraction technique.

Audio-Visual distraction technique was chosen because it is a very simple technique with low cost that can be used by any dentist without requiring specific skills. It is also applicable on almost all patients even adults that could be quiet entertaining for patients and motivating them to attend other future dental visits. Ram et al., 2010 stated that AV distraction using eyeglasses showed better effect than regular television and also it could be used instead of nitrous oxide sedation for decreasing dental anxiety and fear during the dental visit (Ram et al., 2010).

The VR eyeglasses used in this study (Nibiru 2.10.002 AV eyewear glasses with screen size 5 inches and 3D optical resin lens) was selected due to its smaller size, lower cost, and complete blocking of the patient's field of vision which overcame the limitations of the eyeglasses used by Al-Khotani et al., where the size of the device was annoying during dental work and it did not completely block the vision away from the child's eye. (Al-Khotani et al., 2016).

Tell-Show-Do (introduced by Addleston in 1959) has been the technique that is most widely used and compared with other behavior management approaches since then, and is still the most used technique all over the world, therefore, it was the technique used in the control group.

This study included children aged between 5 to 8 years old. According to Piaget's classification, children above the age of 5 , show better recognition and acceptance of the surrounding environment, moreover they also show better attention, concentration and social communications (Afshar et al., 2011).

It was mentioned that children show more distress and anxiety when the dental visit exceeds the duration of 30 minutes. Therefore, in this study the duration of the visit was 20 minutes and the patient was allowed to complete his cartoon show if it was not yet finished (Attar \& Baghdadi, 2014).

Participants had no previous dental experience to assure that the patient does not suffer from any previous psychological trauma and assure that the results calculated are equal among all participants because it was mentioned in a study conducted by Azami et al., that patients with previous dental visits show higher level of anxiety and fear than patients without any previous dental visits (Azami-Aghdash et al., 2015).

The study included patients with dental decay requiring pulp therapy and excluded cases with irreversible pulpitis or chronic pulpal disease in order to avoid any pain response that can occur after administration of the local anesthetic agent and assure same anesthetic and pain stimulation to all patients. 
Pulse oximeter was used to record the patient's heart rate per minute before, after and during the dental visit, since anxiety can result in physiological changes like releasing of certain hormones as adrenaline and increasing of respiratory rate and heart rate. It was important to assess the pulse rate before any dental procedures to record the child's baseline heart rate. And during the dental visit it was recorded to asses if the management protocol was doing any good or harm for the anxiety level. Also by the end of the visit the last record gave a general idea if the child enjoyed the visit and will further attend future dental visit or not (Harrison et al., 2015).

The results of pulse rate readings in this study showed no statistically significant difference between both groups at the initial readings. This helped in standardization between the two groups.

In the control group, after injection of local anesthesia there was a significant difference in the results of pulse rate readings between the initial readings and readings at local anesthetic injection, after 5 minutes and after 10 minutes of the procedure. The heart rate was higher which elaborates that the child was in a stressful and anxious emotional state from the time of injection of the dental needle till after 10 minutes duration. While after 15 and 20 minutes, there was no significant difference from the initial reading which means that the child was more relaxed later after the middle of the procedure.

In the AV eyewear group, results showed that there was no statistically significant difference in heart rate readings during the procedure time. This proves that the child was more relaxed during the procedure especially after local anesthetic injection and the first 10 minutes of the procedure. A similar result was noticed in the study performed by ElSharkawi et al, where he measured the effect of AV distraction with local anaesthesia (El-Sharkawi et al., 2012).

Regarding pulse rate, some studies showed similar results when the pulse rate was measured as an assessment of anxiety level when using audiovisual distraction eyeglasses. In the study conducted by Prabhakar et al. (2007), Mitrakul et al. (2015), and Kaur et al. (2015), AV eyeglasses effectively reduced heart rate during the dental visit. While the studies conducted by Nuvvula et al. (2015), Al-Khotani et al. (2016), and Garrocho et al. (2018), showed no statistically significant difference between AV group and control group

Virtual Reality sickness is a well-known phenomenon, where some individuals suffer motion sickness from computer-generated virtual environments. In this study two individuals from the intervention group out of twenty-one suffered virtual reality sickness as nausea, sweating and blurred vision and were excluded from the study (Dużmańska et al., 2018).

Among the limitations of this study, the size of the eyeglasses was relatively large, although the type used was among the smallest in size. Virtual reality sickness is also considered one of the limitations that can affect the results of studies using AV distraction. In addition to the need to assess other parameters than heart rate.

\section{CONCLUSIONS}

Audiovisual distraction can be considered an effective method for reducing anxiety during dental treatment and helping the patients enjoy the dental visit.

\section{REFERENCES}

1. AAPD (2019) Behavior Guidance for the Pediatric Dental Patient: American Academy of Pediatric Dentistry, 40 (6)

2. Addleston HK. (1959) Child patient training: Fort Rev Chicago Dent Soc, 38:7-9, 27-29.

3. Afshar, H., Baradaran Nakhjavani, Y., Mahmoudi-Gharaei, J., Paryab, M. \& Zadhoosh, S. (2011) The Effect of Parental Presence on the 5 year-Old Children's Anxiety and Cooperative Behavior in the First and Second Dental Visit: Iranian journal of pediatrics, 21 (2), pp.193-200. 
4. Al-Khotani, A., Bello, L.A. \& Christidis, N. (2016) Effects of audiovisual distraction on children's behaviour during dental treatment: a randomized controlled clinical trial: Acta odontologica Scandinavica Journal,74 (6), pp.494-501.

5. Aminabadi, N.A., Erfanparast, L., Sohrabi, A., Oskouei, S.G., Naghili, A. (2012) The Impact of Virtual Reality Distraction on Pain and Anxiety during Dental Treatment in 4-6 Year-Old Children: a Randomized Controlled Clinical Trial: Journal of Dental Research Dental Clinic Dental Prospects, 6(4), pp. 117-124

6. Attar, R.H. \& Baghdadi, Z.D. (2015) Comparative efficacy of active and passive distraction during restorative treatment in children using an iPad versus audiovisual eyeglasses: a randomised controlled trial: Journal of the European Academy of Paediatric Dentistry, 16 (1), pp.1-8.

7. Ai Aghdash, S., Ebadifard Azar, F., Pournaghi Azar, F., Rezapour, A., Moradi-Joo, M., Moosavi, A. \& Ghertasi Oskouei, S. (2015) Prevalence, etiology, and types of dental trauma in children and adolescents: Systematic review and meta-analysis: Medical Journal of the Islaamic Repubublic of Iran, 29:234.

8. Carter, A.E., Carter, G., Boschen, M., AlShwaimi, E. \& George, R. (2014) Pathways of fear and anxiety in dentistry: A review: World journal of clinical cases, 2 (11), pp.642-653.

9. Dużmańska, N., Strojny, P. \& Strojny, A. (2018) Can Simulator Sickness Be Avoided? A Review on Temporal Aspects of Simulator Sickness: Frontiers in psychology, 9, p.2132.

10. El-Sharkawi, H.F.A., El-Housseiny, A.A. \& Aly, A.M. (2012) Effectiveness of new distraction technique on pain associated with injection of local anesthesia for children: Journal of Pediatric dentistry, 34 (2), pp. 35-8.

11. Facco, E. \& Zanette, G. (2017) The Odyssey of Dental Anxiety: From Prehistory to the Present. A Narrative Review: Frontiers in psychology, 8, p.1155.

12. Garrocho-Rangel A, Ibarra-Gutiérrez E, Rosales-Bérber M, Esquivel- Hernández R, Esparza-Villalpando V, PozosGuillén A. (2018) A video eyeglasses/earphones system as distracting method during dental treatment in children: A crossover randomised and controlled clinical trial: European Journal of Pediatric Dentistry, 19(1): 74-79.

13. Goulart, J.C., Pinheiro, M.D., Rodrigues, R.V., Santos, F. (2011) Influence of anxiety on blood pressure and heart rate during dental treatment. Revista Odonto Ciência, 27(1), pp.31-35

14. Grupe, D.W. \& Nitschke, J.B. (2013) Uncertainty and anticipation in anxiety: an integrated neurobiological and psychological perspective: Nature reviews: Neuroscience, 14 (7), pp.488-501.

15. Gupta, A., Marya, C.M., Bhatia, H.P. \& Dahiya, V. (2014) Behaviour management of an anxious child: Stomatologija, 16 (1), pp.3-6.

16. Harrison, D., Bueno, M. \& Reszel, J. (2015) Prevention and management of pain and stress in the neonate: Research and Reports in Neonatology, p.9.

17. Kaur, R., Jindal, R., Dua, R., Mahajan, S., Sethi, K. \& Garg, S. (2015) Comparative evaluation of the effectiveness of audio and audiovisual distraction aids in the management of anxious pediatric dental patients: Journal of the Indian Society of Pedodontics and Preventive Dentistry, 33 (3), pp.192-203.

18. Liu, Y., Gu, Z., Wang, Y., Wu, Q., Chen, V., Xu, X. \& Zhou, X. (2019) Effect of audiovisual distraction on the management of dental anxiety in children: A systematic review: International Journal of Paediatric Dentistry, 29 (1), pp.14-21.

19. Mitrakul, K., Asvanund, Y., Arunakul, M. \& PakaAkekaphat, S. (2015) Effect of audiovisual eyeglasses during dental treatment in 5-8 year-old children: European journal of paediatric dentistry, 16 (3), pp.239-245.

20. Muppa, R., Bhupatiraju, P., Duddu, M., Penumatsa, N.V., Dandempally, A. \& Panthula, P. (2013) Comparison of anxiety levels associated with noise in the dental clinic among children of age group 6-15 years: Noise \& health, 15 (64), pp.190-193.

21. Nuvvula, S., Alahari, S., Kamatham, R. \& Challa, R.R. (2015) Effect of audiovisual distraction with 3D video glasses on dental anxiety of children experiencing administration of local analgesia: a randomised clinical trial: European archives of paediatric dentistry, 16 (1), pp.43-50.

22. Prabhakar, A.R., Marwah, N. \& Raju, O.S. (2007) A comparison between audio and audiovisual distraction techniques in managing anxious pediatric dental patients: Journal of the Indian Society of Pedodontics and Preventive Dentistry, 25 (4), pp.177-182. 
23. Ram, D., Shapira, J., Holan, G., Magora, F., Cohen, S. \& Davidovich, E. (2010) Audiovisual video eyeglass distraction during dental treatment in children: Quintessence international, 41 (8), pp.673-679.

24. Vilela, R.C.I.C.R.J.E.R. \& Ogawa, M.S.R.W.N. (2019) Effect of awards after dental care in children's motivation: European Archives of Paediatric Dentistry, 20 (2), pp.85-93.
25. Wang, Z.-X., Sun, L.-H. \& Chen, A.-P. (2008) The efficacy of non-pharmacological methods of pain management in school-age children receiving venepuncture in a paediatric department: a randomized controlled trial of audiovisual distraction and routine psychological intervention: Swiss medical weekly, 138 (39-40), pp.579-584. 